\title{
Light-Matter Quantum Interface
}

\author{
K. Hammerer ${ }^{1}$, K. Mølmer ${ }^{2,3}$, E.S. Polzik ${ }^{2,4}$, J.I. Cirac ${ }^{1}$ \\ ${ }^{1}$ Max-Planck-Institut für Quantenoptik, Hans-Kopfermann-Strasse, D-85748 Garching, Germany \\ 2 QUANTOP, Danish Research Foundation Center for Quantum Optics \\ 3 Department of Physics and Astronomy, University of Aarhus, DK 8000 Aarhus C, Denmark \\ 4 Niels Bohr Institute, DK 2100 Copenhagen, Denmark
}

\begin{abstract}
We propose a quantum interface which applies multiple passes of a pulse of light through an atomic sample with phase/polarization rotations in between the passes. Our proposal does not require nonclassical light input or measurements on the system, and it predicts rapidly growing entanglement of light and atoms from just coherent inputs. The proposed interface makes it possible to achieve a number of tasks within quantum information processing including teleportation between light and atoms, quantum memory for light and squeezing of atomic and light variables.
\end{abstract}

PACS numbers: 03.67.Mn, 32.80.Qk

Quantum networks require an efficient quantum interface between light, which is a natural long distance carrier of quantum information, and atoms, that make a better storage and processing medium. The power of such a device will be intimately connected to its capability of creating high degrees of entanglement in a controlled way, since entanglement represents an all-purpose resource to create conditional dynamics.

Numerous theoretical and experimental works ([1] and references therein) center around the effect of a Kerr interaction between light and atomic ensembles to produce entanglement between continuous light-atom variables. This interspecies entanglement can in turn be converted to atomic spin correlations in form of spin squeezed or spin entangled states between two atomic samples [2] by means of a projection measurement on light. If spontaneous emission is neglected, the degree of squeezing is of the order of $\Delta=1 /(1+\kappa)$ where $\kappa$ is the effective coupling strength between light and atoms. Thus, it seems that one can, in principle, produce in this way unlimited atomic squeezing. There are however serious limitations on both, the amount of light atom entanglement as well as the degree of squeezing, which can be achieved from a Kerr interaction. In fact one can express $\kappa=\alpha_{0} \eta$ where $\alpha_{0}$ is the sample's optical (column) density and $\eta$ is the spontaneous emission probability. Thus, decoherence due to spontaneous emission cannot be neglected and a crude estimate for $\eta \ll 1$ leads to an additional contribution, so that $\Delta=1 /\left(1+\alpha_{0} \eta\right)+2 \eta(\Delta=1$ corresponds to a coherent state). Apparently this expression has a minimum $\Delta_{\min }=2 \sqrt{2 / \alpha_{0}}$ corresponding to an optimal spontaneous decay probability $\eta_{0}=1 / \sqrt{2 \alpha_{0}}$. In realistic systems the optical density is often limited to values in the range between 1-100, which is true for atomic vapors (ref. [7]), as well as for cold and trapped atoms. For the optical density of, say, 25 , the above estimates lead to the limit on squeezing of the order of $\Delta_{\min } \approx 0.5(3 \mathrm{~dB}$ of noise reduction) with a single pass QND measurement. The same consideration limits also the amount of light atom entanglement present before the measurement (see
Fig.1).

But there is still another peculiarity of the Kerr interaction, which limits its performance in creating entanglement: Due to its QND character this interaction conserves certain degrees of freedom, which is reflected in a strict limit on the amount of achievable EPR-type squeezing to $\triangle E P R=0.5$. Thus, even for an arbitrarily high optical density, the state which originates from a Kerr interaction is never close to a maximally entangled EPR state corresponding to $\triangle E P R \rightarrow 0$.

In this Letter we propose experimentally feasible techniques which allow to overcome these limitations and in fact provide an exponential growth in the amount of entanglement and squeezing. Two and three pass protocols have already been proposed in $[1,4,5]$. Taking spontaneous decay into account, we show that passing one and the same pulse of light $n$ times through an atomic ensemble produces an effective optical density of $n \alpha_{0}$ while the effect of accumulated spontaneous emission noise can be balanced by tuning $\eta$ to its optimal value for a given number of steps $n$. Hence, although the coupling strength in a Kerr interaction is directly proportional to the probability of spontaneous decay, this does not pose a fundamental limit on the generation of entanglement or squeezing.

Based on this result we show furthermore that this system provides the realistic possibility to implement a quantum information algorithm proposed in [3] for generating entanglement and squeezing at optimal rates in pure Gaussian continuous variable states. This algorithm is based on the idea to intersperse interactions with local operations and to optimize these local operations such as to maximally increase the quantity of interest. In the system under consideration these optimal local operations can be effected simply by $\lambda / 4$ plates and mirrors changing the polarization and direction of light propagation in between the passes of the atomic sample. We determine for realistic experimental parameters the optimal spontaneous decay probability for a given number of steps and show thereby that even in the presence of losses the growth of entanglement is still significantly enhanced. In 
particular, one can in this way engineer a state which is close to an EPR state.

Finally we also suggest a way to convert the entanglement unconditionally into squeezing of the atoms without the use of homodyne detection of light. This method which relies on a certain choice of polarization rotations is as powerful as the QND measurement and it yields in addition a squeezed optical output.

The system we are considering is the same as in $[2],[6],[7]$. It is shown there that the interaction between an off-resonant laser pulse and an ensemble of atoms with total angular momentum equal to $\hbar / 2$ is appropriately described by a Kerr-interaction Hamiltonian $H \propto J_{z} S_{z}$ with collective atomic spin operator $J_{z}=\frac{1}{2} \sum_{i} \sigma_{z}^{(i)}$ and Stokes operator $S_{z}=\left(a_{R}^{\dagger} a_{R}-a_{L}^{\dagger} a_{L}\right) / 2$. Here $\sigma_{z}^{(i)}$ is the Pauli spin operator along $z$ for the $i$-th atom and $a_{R}\left(a_{L}\right)$ is the photon annihilation operator for right (left) circularly polarized photons. In the limit of large polarization along the $x$-direction one can treat $J_{x}$ and $S_{x}$ as classical variables and represent the orthogonal directions by canonical operators $x_{a t}=J_{y} / \sqrt{\left\langle J_{x}\right\rangle}, p_{a t}=$ $J_{z} / \sqrt{\left\langle J_{x}\right\rangle}, x_{p h}=S_{y} / \sqrt{\left\langle S_{x}\right\rangle}, p_{p h}=S_{z} / \sqrt{\left\langle S_{x}\right\rangle}$. Both the atomic coherent spin state and the polarized laser pulse correspond in this limit to the ground states of the harmonic oscillators associated with these operators. The interaction Hamiltonian in the oscillator formulation becomes $H \propto \sqrt{\left\langle J_{x}\right\rangle\left\langle S_{x}\right\rangle} p_{a t} p_{p h}$. Since the initial states are Gaussian distributions over the phase space and the Hamiltonian is bilinear in the canonical operators and therefore conserves the Gaussian character of the state it is appropriate to express the dynamics in the Schrödinger picture in terms of a displacement vector $\vec{d}=\operatorname{tr}\{\vec{R} \rho\}$ and a covariance matrix $\gamma_{i, j}=\operatorname{tr}\left\{\rho\left[\left(\vec{R}_{i}-\vec{d}_{i}\right),\left(\vec{R}_{j}-\vec{d}_{j}\right)\right]_{+}\right\} \quad(i, j=1, \ldots, 4)$ where $\vec{R}=\left(x_{a t}, p_{a t}, x_{p h}, p_{p h}\right)$ and $[., .]_{+}$denotes the anticommutator. For the given initial states and within the above approximation we have $\vec{d}=\overrightarrow{0}$ for all times. Thus, all information about the compound system can be extracted from its covariance matrix.

The state after a single pass of a pulse of light through the atomic ensemble is described in terms of input-output relations as

$$
\gamma_{\text {out }}=\bar{D}(\eta, \epsilon) S(\kappa) \gamma_{\text {in }} S(\kappa)^{T} \bar{D}(\eta, \epsilon)+D(\eta, \epsilon) \gamma_{\text {noise }}
$$

where the scattering matrix

$$
S(\kappa)=\left(\begin{array}{llll}
1 & 0 & 0 & \kappa \\
0 & 1 & 0 & 0 \\
0 & \kappa & 1 & 0 \\
0 & 0 & 0 & 1
\end{array}\right)
$$

and $D(\eta, \epsilon)=\operatorname{diag}(\eta, \eta, \epsilon, \epsilon), \quad \bar{D}(\eta, \epsilon)=$ $\sqrt{1-D(\eta, \epsilon)}, \quad \gamma_{\text {noise }}=\operatorname{diag}(2,2,1,1)$. The output state is a weighted sum of a coherent contribution and a noise component $\gamma_{\text {noise }}$ whose form is due to the fact that the field decay is accompanied by a vacuum noise contribution and the atomic decay both contributes to noise due to the breaking of correlations among the atoms and due to the atoms once decayed being still present in the sample, explaining the factor of 2 in the atomic component of $\gamma_{\text {noise }}$. Apart from this correction Eq. (1) is equivalent to the result derived in [7]. In principle, the noise introduced in atoms increases with the decay of the mean polarization, but this effect is negligible for the example presented (see [8] for a refined model for this interaction using the same formalism).

The coupling constant is given by $\kappa=$ $2 \sqrt{\left\langle J_{x}\right\rangle\left\langle S_{x}\right\rangle} \sigma \Gamma / A \Delta$, the atomic depumping $\eta=N_{p h} \sigma \Gamma^{2} / A \Delta^{2}$ and the photonic absorption rate $\epsilon=N_{a t} \sigma \Gamma^{2} / A \Delta^{2}$ where $\sigma$ is the cross section on resonance for the probed transition, $\Gamma$ is the corresponding spontaneous decay rate, $\Delta$ the detuning from resonance and $A$ the cross section of the atomic ensemble illuminated by the pulse. Equation (1) is valid for small atomic dephasing and low photon absorption corresponding to $\eta, \epsilon \ll 1$.

A central quantity in this system is the optical density on resonance $\alpha_{0}=N_{a t} \sigma / A$ which gives the probability for a single photon to get elastically scattered and can be related to the other parameters as $\epsilon=\alpha_{0}(\Gamma / \Delta)^{2}$ and $\kappa^{2}=\eta \alpha_{0}$ where we used that initially $\left\langle J_{x}\right\rangle=N_{a t} / 2$ and $\left\langle S_{x}\right\rangle=N_{p h} / 2$. There is an apparent tradeoff between having a large coupling and at the same time low atomic depumping. For a given optical density one can treat $\epsilon$ and $\eta$ as independent parameters tailoring the first by means of the detuning and the last by means of $N_{p h}$, and there are always optimal values for $\epsilon$ and $\eta$ which maximize the achievable squeezing or entanglement.

We are here especially interested in three quantities characterizing the quantum properties of the state generated: (a) the Gaussian Entanglement of Formation (GEOF) [9], the only available physical Entanglement measure for mixed Gaussian bipartite states, (b) the closely related [10] EPR uncertainty of the combined atom+field system, which indicates how close the state is to a maximally entangled EPR state, given for the present states by $\Delta E P R=\frac{1}{2}\left[\Delta^{2}\left(x_{a t}-p_{p h}\right)+\Delta^{2}\left(p_{a t}-\right.\right.$ $\left.\left.x_{p h}\right)\right]$, and finally (c) the atomic (and light) squeezing achievable either by a QND measurement (homodyne detection of light) or by means of a particular disentangling operation at the end of the multi pass protocol.

The state created after several passes can be calculated by iterating the map defined by equation (1). Note however that the coupling strength depends on the polarizations along $x$ and that these classical variables will decay from pass to pass as $\left\langle J_{x}\right\rangle_{\text {out }}=(1-\eta)\left\langle J_{x}\right\rangle_{\text {in }},\left\langle S_{x}\right\rangle_{\text {out }}=$ $(1-\epsilon)\left\langle S_{x}\right\rangle_{i n}$. For the $n$-th step the remaining coupling strength is hence reduced $\kappa_{n}=[(1-\eta)(1-\epsilon)]^{n / 2} \kappa$. Reflection losses can be taken into account by replacing $\epsilon$ by $\zeta=\epsilon+r$ where $r$ is the overall reflectivity of mirrors, cell etc. Equation (1) provides then readily a recursion 

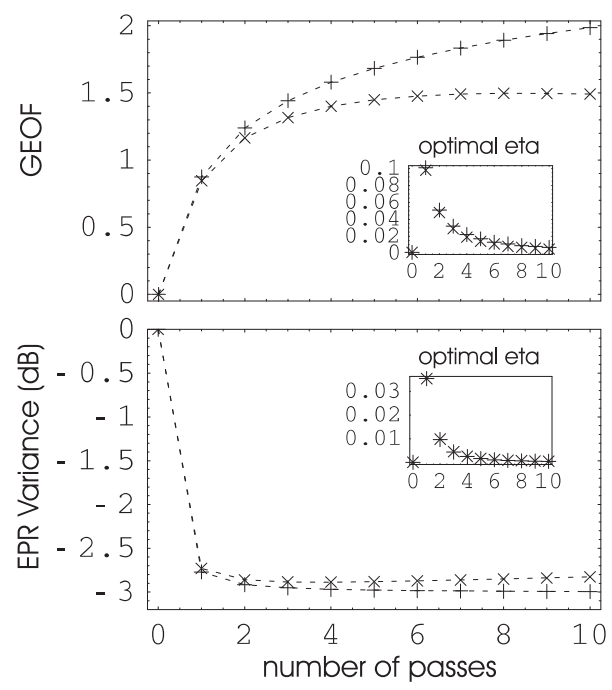

FIG. 1: GEOF and EPR variance vs. number of passes: For given $n$ both quantities are maximized with respect to $\eta$ and $\zeta$. The optimal values for $\eta$ are shown in the inserts. It is always best to have $\zeta=r$ corresponding to $\epsilon \ll \eta$. +'es refer to the case $r=0, \times^{\prime}$ es to $r=2 \%$. The optical density is $\alpha_{0}=25$.

relation

$$
\gamma_{n}=\bar{D}(\eta, \zeta) S\left(\kappa_{n}\right) \gamma_{n-1} S\left(\kappa_{n}\right)^{T} \bar{D}(\eta, \zeta)+D(\eta, \zeta) \gamma_{n o i s e}
$$

for the state after $n$ passes which can be solved exactly.

The effect of $n$ consecutive passes is comparable to that of a single pass performed with an $n$ times increased optical density. This is clear from the meaning of $\alpha_{0}$ and becomes manifest in the group property $S(\kappa) S(\lambda)=$ $S(\kappa+\lambda)$ of the scattering matrix (2). This indicates that the strategy of multiple passes is especially interesting for low optical densities. The dependence of the GEOF and the EPR variance on the number of passes is shown in figure 1. In general it can be shown under the assumption of vanishing reflection losses $(r=0)$ that for given optical density and number of steps $n$ there exist optimal choices for $\eta$ and $\epsilon$ such that, taking formally $n \rightarrow \infty$, the GEOF tends to infinity. The EPR-variance is limited by 0.5 , or $3 \mathrm{~dB}$ of squeezing, which is also evident in figure 1.

The multipass scheme is capable of improving these features significantly. In particular, applying a unitary operation and its adjoint before and after an interaction changes effectively the type of interaction due to the identity $U^{\dagger} \exp (-i H) U=\exp \left(-i U^{\dagger} H U\right)$. The transformations which are easy to perform in this system are polarization rotations which change the quadratures as $x \rightarrow \cos \phi x+\sin \phi p, p \rightarrow \cos \phi p-\sin \phi x$. In [3] it was shown in a pure state analysis that entanglement and squeezing is created at a maximal rate if one switches from $H \propto p_{a t} p_{p h}$ to an interaction $H \propto-x_{a t} x_{p h}$ in every second step. The effect of the switching becomes clear if one approximates $\exp \left(i x_{a t} x_{p h} \kappa\right) \exp \left(-i p_{a t} p_{p h} \kappa\right) \simeq$

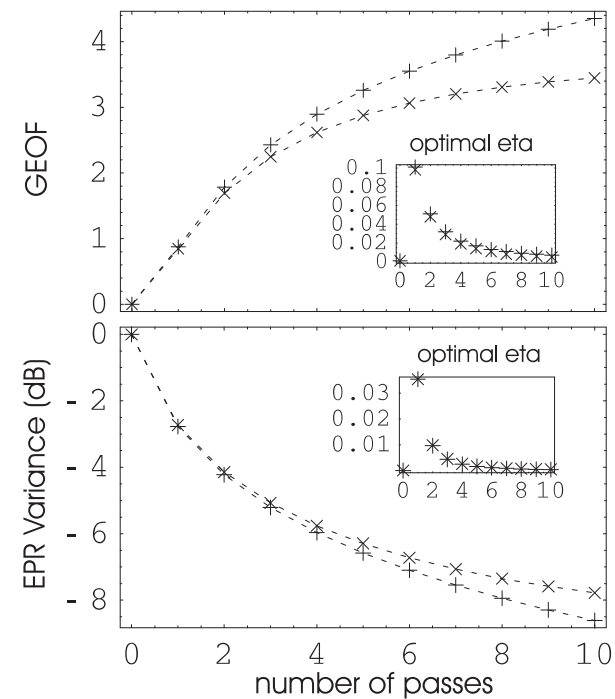

FIG. 2: GEOF and EPR variance vs. number of passes including polarization rotations: ${ }^{\prime}$ 'es refer to the case $r=0$, $\times^{\prime}$ es to $r=2 \%$. Optical density $\alpha_{0}=25$.

$\exp \left[-i\left(p_{a t} p_{p h}-x_{a t} x_{p h}\right) \kappa+o\left(\kappa^{2}\right)\right]$. To first order this interaction creates a two-mode squeezed state. In particular the growth is linear in $n$ and thus provides an exponential improvement as compared to the scheme without switching. The final state after $n$ passes follows from equation (3) by taking the scattering matrix to be $S(\kappa)^{T}$ - corresponding to an interaction $H \propto-x_{a t} x_{p h}$ - in every second step. Figure 2 shows how the quantities of interest develop. In comparison with the unswitched case, the GEOF is roughly doubled and the EPR squeezing is no longer limited to $3 \mathrm{~dB}$. In the limit of $n \rightarrow \infty$ the resulting state approximates a maximally entangled EPR state which can be used as a resource for continuous variable teleportation. This provides an attractive possibility to establish a quantum memory for light since an unknown quantum state of light can be teleported onto the atoms by performing a joint measurement on the unknown input state and the optical component of the EPR state.

After multiple passes (with or without switching of polarizations) neither light nor atomic quadratures are squeezed separately. In order to obtain such local squeezing an additional operation has to be carried out. One possibility is to perform a destructive homodyne detection of light, which - in the unswitched scheme - amounts to a QND measurement of the atomic $p$-quadrature and yields a squeezed state of atoms while the light is lost. Performing the same measurement on one half of an EPR state - as it arises in the switched scheme - also leaves the other system in a squeezed state. Figure 3 displays the atomic squeezing after a homodyne detection of light for both schemes. The switching provides a small advantage even though the actual interaction has lost its QND character. The tradeoff between squeezing and spontaneous emission noise has also been discussed in [11] for a 


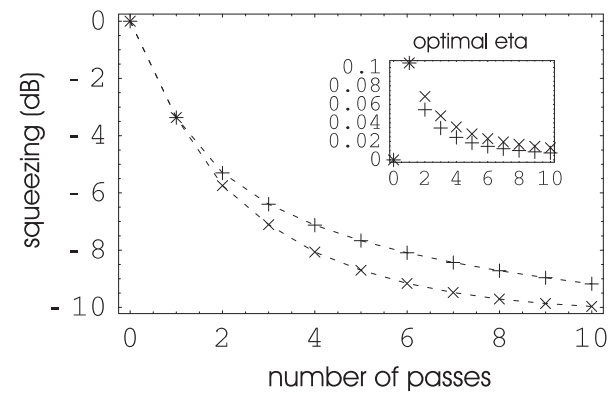

FIG. 3: Atomic squeezing after homodyne detection of light: Unswitched scheme (QND measurement) " + " and switched scheme $" \times " . \zeta=r=2 \%, \alpha_{0}=25$.

\section{different type of interaction.}

We now show that provided the coupling strength $\kappa$ can be tuned to a certain value, it is possible to disentangle the state created after several passes by an appropriate last passage of the light pulse through the atomic cloud. The basic mechanism is most clearly seen on the basis of pure states and for the scheme without polarization switching, but it can be easily adapted also for the other case. After $n$ passes the atom and field operators have evolved in the Heisenberg picture as given by $\vec{R}^{\prime}=S(n \kappa) \vec{R}_{\text {in }}$ where $\vec{R}$ is defined as above. By switching to an interaction $H \propto x_{a t} x_{p h}$ a single additional pass then yields a state $\vec{R}_{\text {out }}=S(-\kappa)^{T} \vec{R}^{\prime}$ and thus $p_{a t}^{o u t}=p_{a t}^{\prime}-\kappa x_{p h}^{\prime}=\left(1-n \kappa^{2}\right) p_{a t}^{i n}-\kappa x_{p h}^{i n}$. For $0<n \kappa^{2} \leq 1$ this last pass reduces the weight factor of $p_{a t}$ indicating the possibility of squeezing but at the same time it feeds light noise into the atomic variance. With initial coherent states one finds $\left\langle\left(p_{a t}^{\text {out }}\right)^{2}\right\rangle=\left[\left(1-n \kappa^{2}\right)^{2}+\kappa^{2}\right] / 2$. This expression can be minimized with respect to the value of $\kappa$, and the optimal value $\kappa_{0}=\sqrt{n-1 / 2} / n$ leads to a squeezing of $\left\langle\left(p_{a t}^{\text {out }}\right)^{2}\right\rangle /\left\langle\left(p_{a t}^{\text {in }}\right)^{2}\right\rangle=1 / n-1 / 4 n^{2}$ in comparison with the value $1 /(n+1 / 2)$ achievable in a QND measurement with the same coupling strength $\kappa_{0}$. For large $n$ the difference between these two expressions is negligible. An important aspect of the decoupling scheme is that it is not conditioned on a measurement result, and as a side benefit, light is actually simultaneously squeezed. Figure 4 shows the squeezing of both light and atomic quadratures after such a disentangling step as well as the result of a comparable QND measurement with identical coupling $\kappa$.

The experimental feasibility of the proposal is illustrated with the following example. Consider an ensemble of cold 87Rb atoms with two ground magnetic states, $F=1, m_{F}= \pm 1$, forming the atomic two-level spin system. The light is coupled to these states via D1 transition (HWHM natural linewidth $2.5 \mathrm{MHz}$ ). Assuming a cylindrical atomic sample with the diameter 100 microns and the length of 500 microns containing $2 \times 10^{6}$ atoms corresponding to a typical dipole trap density of $5 \times 10^{11} \mathrm{~cm}^{-3}$, a resonant optical density of 25 can be achieved with the

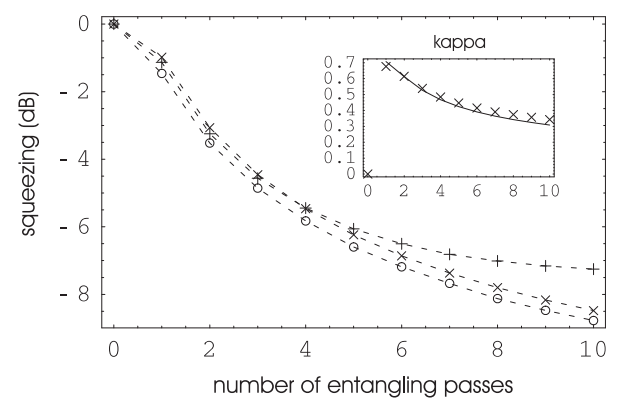

FIG. 4: Squeezing of light ("+") and atomic (" $\times ")$ quadrature after $n$ entangling and a single disentangling step. Result from a comparable QND measurement on the atomic system "०". $\zeta=r=2 \%, \alpha_{0}=25$. Insert: Optimal value for coupling $\kappa_{\text {opt }}=\alpha_{0} \eta_{\text {opt }}$ and theoretical magical value $\kappa_{0}=$ $\sqrt{n-1 / 2} / n$ (solid line). Atomic depumping $\eta$ decreases with $\kappa$ while light suffers a constant loss of $2 \%$ per pass. Therefore the asymmetry in squeezing of light an atomic variables.

atomic dipole crossection $\sigma=10^{-9} \mathrm{~cm}^{2}$. To meet the optimal condition of light absorption being much less than the spontaneous emission probability, $\epsilon \ll \eta$, we choose the light detuning greater than $100 \mathrm{MHz}$. Then $\epsilon$ is reduced to less than $1.5 \times 10^{-3}$. Since $\eta / \epsilon=N_{p h} / N_{a t}$, we can now adjust the optimal value for $\eta$ found from theoretical graphs in Fig. 1-4 by choosing the optimal number of photons per pulse. For $\eta$ in the range of $0.01-0.1$ the optimal photon number per pulse is $10^{7}-10^{8}$. This number of photons is close to optimal for shot noise limited balanced detection. In order to fit the experiment on a table top the physical length of light pulses should not exceed a few meters, since the "tail" of the pulse should clear through the sample before its "head" enters the sample in the next pass. A $3 m$ pulse length corresponds to about $30 \mathrm{MHz}$ Fourier limited bandwidth which fits well with the detuning somewhat greater than $100 \mathrm{MHz}$. Switching of the interaction from X-type to Ptype for light can be achieved simply by passing the light through a $\lambda / 4$ plate in between the passes. For atoms this switching can be achieved by changing the propagation direction of light by 90 degrees.

In summary, we have proposed a quantum interface between light and atoms capable of performing valuable tasks in quantum information processing. By means of several interaction steps and local operations it is possible to efficiently create entangled and squeezed states. In particular one can engineer an EPR state which can act as a resource for a quantum memory for light. Furthermore we showed that without performing any measurement our multipass scheme allows to create at the same time spin squeezed atoms and quadrature squeezed light.

This work was supported by the EU IST project RESQ, the EU grant QUICOV and the Kompetenznetzwerk Quanteninformationsverarbeitung der Bayerischen Staatsregierung. 
[1] A. Kuzmich and E.S. Polzik, in Quantum Information with Continuous Variables, S. Braunstein and A. Pati eds. (Kluwer, New York, 2003)

[2] B. Julsgaard, A. Kozhekin, and E. S. Polzik, Nature 413, 400 (2000)

[3] B. Kraus, K. Hammerer, G. Giedke, J.I. Cirac, Phys. Rev. A 67, 042314 (2003)

[4] A. Kuzmich, L.-M. Duan, unpublished

[5] J. Fiurasek, Phys. Rev. A 68, 022304 (2003)
[6] A. Kuzmich, N.P. Bigelow, L. Mandel, Europhys. Let. 42, 481 (1998)

[7] L.-M. Duan, J.I. Cirac, P. Zoller, E.S. Polzik, Phys. Rev. Let. 85, 5643 (2000)

[8] L.B. Madsen, K. Mølmer, quant-ph/0406146

[9] M.M. Wolf, G. Giedke, O. Krüger, R.F. Werner, J.I. Cirac, quant-ph/0306177

[10] G. Giedke, M.M. Wolf, O. Krüger, R.F. Werner, J.I. Cirac, Phys. Rev. Let 91107901 (2003)

[11] A. Andre, M.D. Lukin, Phys. Rev. A 65, 053819 (2002) 